\title{
Utility of brain parenchyma density measurement and computed tomography perfusion imaging in predicting brain death
}

\author{
Asli I. Akdogan', Hilal Sahin², Yeliz Pekcevik², Hatice Uluer ${ }^{3}$ \\ 'Department of Radiology, Buca Women Birth and Child Diseases Hospital, Izmir, Turkey \\ ${ }^{2}$ Department of Radiology, University of Health Sciences, Tepecik Training and Research Hospital, Izmir, Turkey \\ ${ }^{3}$ Department of Biostatistics, Ege University, Faculty of Medicine, Izmir, Turkey
}

\section{Abstract}

Purpose: To assess the utility of brain parenchyma density measurement on unenhanced computed tomography (CT) in predicting brain death (BD), in order to evaluate the added value of CT perfusion (CTP).

Material and methods: A total of 77 patients who were clinically diagnosed as $\mathrm{BD}$ and had both CT angiography (CTA) and CTP imaging in the same session were retrospectively reviewed. On unenhanced phase of CTA, density measurement was performed from 23 regions of interests (ROIs) which were located in the following areas: level of basal ganglia (caudate nucleus, putamen, corpus callosum, posterior limb of internal capsule), level of brainstem, greywhite matters on levels of centrum semiovale (CS), high convexity (HC), and cerebellum. CTP images were evaluated qualitatively and independently. Grey matter (GM), white matter (WM), density, and GM/WM density ratio of BD patients were compared with control subjects.

Results: Comparing with the normal control group, the GM and WM density at each level and GM/WM density ratio of CS, HC, and cerebellum level were significantly lower in brain-dead patients ( $p=0.019$ for HC-WM, $p<0.001$ for other areas). Using ROC analysis, the highest value of area under curve (AUC) for the GM/WM density ratio was found at the $\mathrm{HC}$ level $(\mathrm{AUC}=0.907)$. The sensitivity of the GM/WM density ratio at the HC level was found to be $90 \%$ when the cut-off value of 1.25 was identified. Evaluating the GM/WM density ratio together with the CTP results increased the sensitivity further to $98 \%$.

Conclusions: The GM/WM density ratio at the HC level on unenhanced CT may be a useful finding to predict BD. Also, the addition of CTP increases the sensitivity of this method.

Key words: brain death, computed tomography angiography, computed tomography perfusion, density.

\section{Introduction}

The algorithm of brain death (BD) diagnosis occurs on the basis of clinical findings and neurological examination, which is followed by ancillary tests, especially when confounding factors (e.g. metabolic and endocrine disturbances, sedative drugs) could affect the clinical evaluation [1] Computed tomography angiography (CTA) has been accepted as one of the ancillary tests and is commonly used for the confirmation of cerebral circulatory arrest in $\mathrm{BD}$ [2].
In addition, computed tomography perfusion (CTP) is known to be a highly sensitive method in the diagnosis of $\mathrm{BD}$ by showing the lack of cerebral perfusion [2-4].

Unenhanced CT findings of BD have been previously described as diffuse cerebral oedema, loss of distinction between grey matter (GM) and white matter (WM), and herniation [5]. Cerebral oedema occurs as a result of increase in water content of GM and is mainly responsible for the reduction of attenuation in the cerebral cortex on CT imaging [6-8]. In two studies conducted by Inamasu et al.,

Correspondence address:

Dr. Asli Irmak Akdogan, Department of Radiology, Buca Women Birth and Child Diseases Hospital, Izmir, Turkey, e-mail: irmakbiranci@gmail.com

Authors' contribution:

A Study design · B Data collection · C Statistical analysis · D Data interpretation · E Manuscript preparation · F Literature search · G Funds collection 
the role of loss of GM-WM discrimination in predicting the prognosis after cardiac arrest was investigated, and it was shown that this sign could be related to poor outcome $[9,10]$. In addition, there are various studies evaluating the prognostic value of the GM/WM ratio as a quantitative method in comatose patients after cardiac arrest [11-17]. According to the American Heart Association guidelines for post-cardiac arrest care, the presence of a significant decrease of GM/WM ratio could be used as a predictor of poor outcome in patients with coma, who are not treated with hypothermia [18].

In routine clinical practice, comatose patients are evaluated cautiously for signs of BD. Nevertheless, it is also critical to have a reliable and accurate method that could predict BD in such patients at early stages, especially for organ donation. For this reason, a few studies have shown the relationship between BD and GM-WM density changes $[19,20]$. However, a study that evaluated unenhanced CT and CTP for the prediction of BD has not been reported, to our knowledge. In this study, we aimed to evaluate the effectiveness of density measurement from various regions on unenhanced CT scan and the contribution of CTP in predicting BD.

\section{Material and methods}

\section{Study population}

The study protocol was approved by our institutional review board. Written informed consent was waived due to the retrospective nature of this study. Between September 2014 and July 2018, 92 patients who underwent CT after clinical diagnosis of $\mathrm{BD}$ were collected from our database. Inclusion criteria were patients with clinically diagnosed $\mathrm{BD}$ and who had CTA and CTP obtained with BD protocol. After the exclusion of nine patients without CTP images, five patients with unenhanced CT images that were unsuitable for density measurement due to the artifacts, and one patient with an extensive extracalvarial herniation after decompressive craniectomy, 77 patients were enrolled in the study. The control group was retrospectively collected from the PACS database, including age- and sex-matched patients who had an unenhanced CT scan without evidence of ischaemia, intracranial haemorrhage, neoplastic lesion, or parenchymal disease. A flow chart of the study is shown in Figure 1.

\section{Computed tomography protocol and density measurements on unenhanced computed tomography}

All CT examinations were acquired with a 128-detector (Siemens Somatom Definition AS, Munich, Germany) or 64-detector (Toshiba Aquilion, Tokio, Japan) CT scanner (64 vs. 13 patients, respectively). The acquisition parameters were slice thickness of $1 \mathrm{~mm}$, pitch $0.55,100 \mathrm{kV}$, $200 \mathrm{mAs}, 220 \mathrm{FOV}$, and $512 \times 512$ matrix in our routine $\mathrm{BD}$ protocol. Despite the fact that this protocol includes two-phase CTA (arterial phase scan at the $20^{\text {th }}$ and venous phase scan at the $60^{\text {th }}$ second after contrast administration), only unenhanced scans were evaluated in this study. CT scans were performed within 2-12 hours of clinical examination of $\mathrm{BD}$. In the control group, unenhanced CT images were obtained with standard head CT protocol with a slice thickness of $5 \mathrm{~mm}$ at either 128-detector or 64-detector CT scanner (67 vs. 10 subjects, respectively). All density measurements of the patient group were performed by two radiologists (H.S. and A.A.) independently, who were blinded to measurements of each other. The measurements of the senior radiologist (H.S.) with 10 years of experience in the assessment of CTA for BD confirmation were accepted as the reference standard. CT images of the control group were measured by the other radiologist (A.A.).

Circular region of interests (ROIs) were placed in several areas in brain parenchyma, which were first described by Torbey et al. [11] and modified by Vigneron et al. [19]. In this study, two different places were added, and Hounsfield unit (HU) measurements were taken from each of 23 ROI in the following areas (Figure 2):

- Level of basal ganglia (BG): caudate nucleus (CN), putamen (PU), corpus callosum (CC), and posterior limb of internal capsule (PIC) were included. The GM to WM ratio was calculated according to the following method, which was described in previous studies [8,12-14]: $\mathrm{GW} / \mathrm{WM}$ density ratio $(\mathrm{BG})=(\mathrm{CN}+\mathrm{PU}) /(\mathrm{CC}+\mathrm{PIC})$.

- Level of centrum semiovale (CS): GM and WM of each hemisphere were measured $5 \mathrm{~mm}$ above the level of BG and average values of $\mathrm{HU}$ were calculated.

- Level of high cerebral convexity (HC): GM and WM of each hemisphere were measured $5 \mathrm{~mm}$ above from level

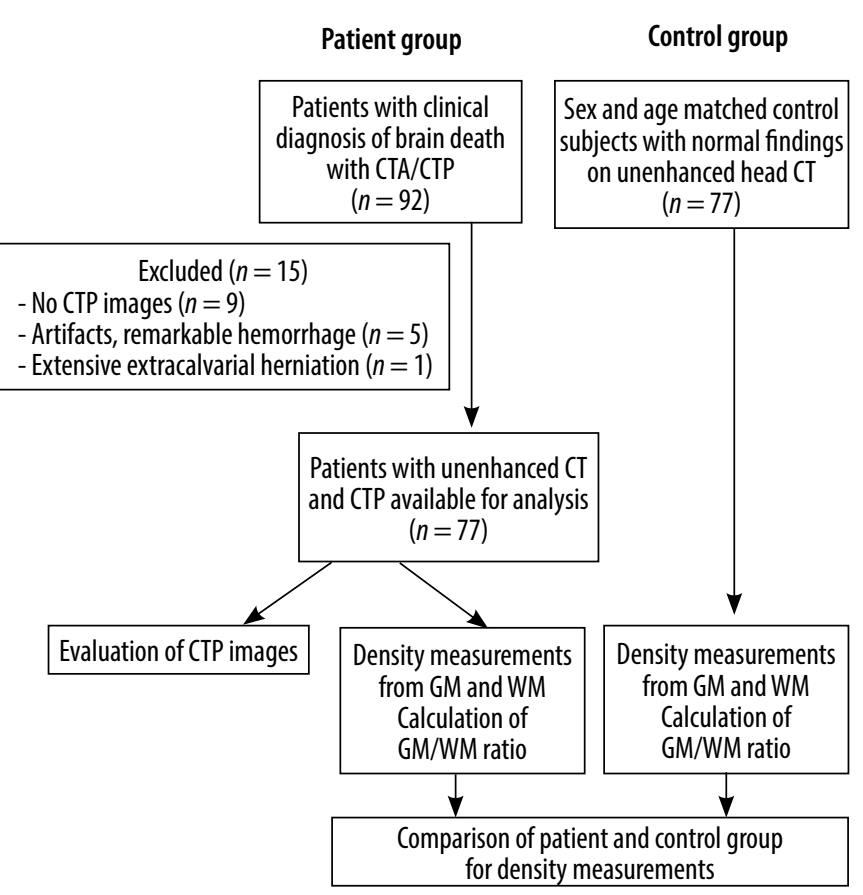

CTA - computed tomography angiography, CTP - computed tomography perfusion, WM - white matter, GM - grey matter

Figure 1. Study flowchart 


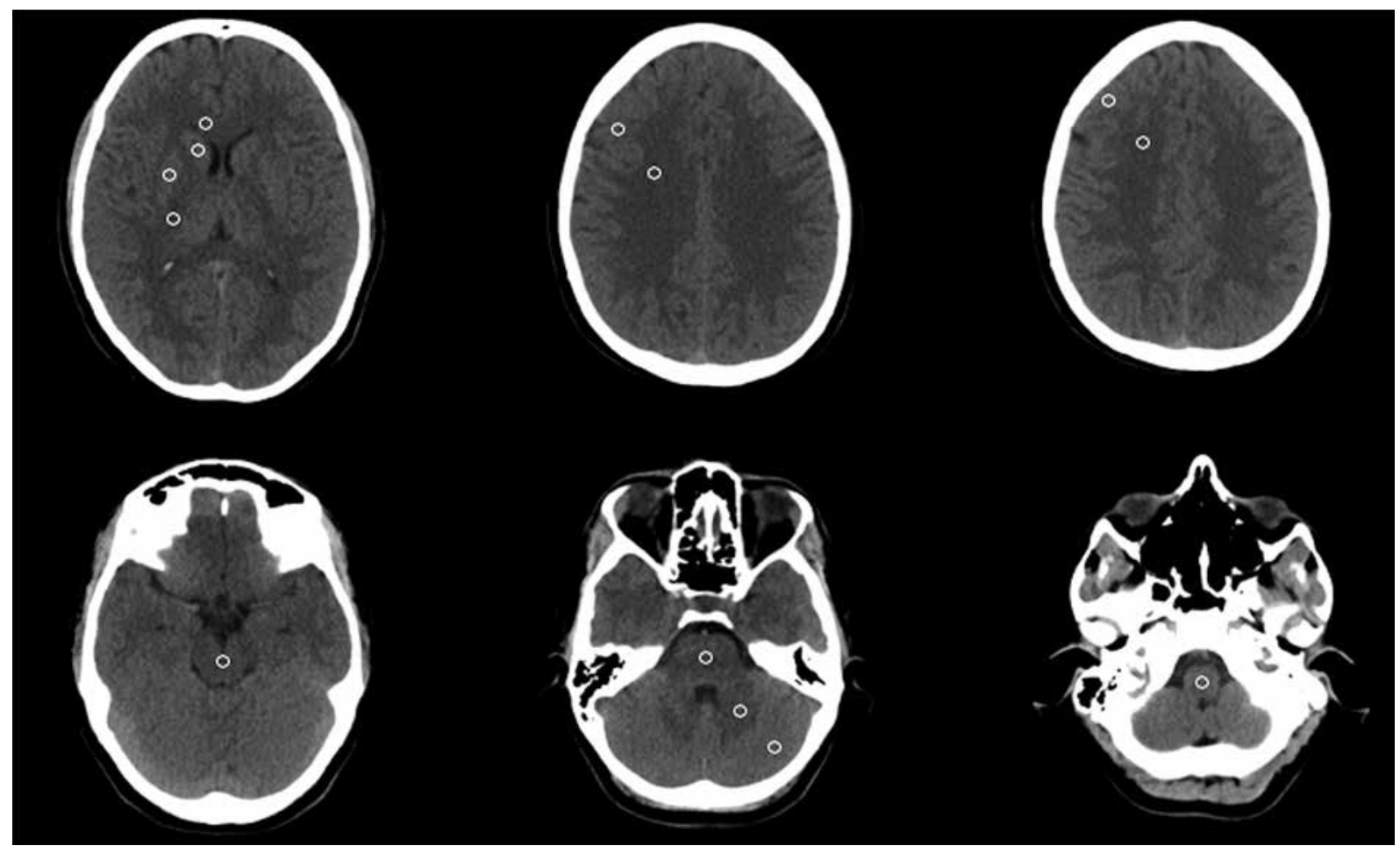

Figure 2. Brain computed tomography images of a normal patient showing the regions where ROl's are placed for the density measurement. From the top left-to the bottom right corner: level of basal ganglia, centrum semiovale, high convexity, midbrain, pons and cerebellum, medulla oblongata, respectively

of CS and averaged. ROIs were placed to medial parts of the brain parenchyma in order not to be affected by beam hardening artifact on both HC and CS levels.

- Level of cerebellum: GM and WM of each hemisphere were measured and averaged.

- Level of brain stem (BS): midbrain, pons, and medulla oblongata were measured from the middle part with an ROI cursor of $20 \mathrm{~mm}^{2}$ differently from other levels, which were calculated with $10 \mathrm{~mm}^{2}$ circular ROI.

In both patient and control groups, the mean $\mathrm{HU}$ values of WM, GM, and GM/WM density ratio were recorded at each level. However, regions that could not be evaluated due to the remarkable haemorrhage, infarct, shift, or artifacts were not included in the analysis in the patient group.

\section{Computed tomography perfusion protocol and interpretation}

CTP images were obtained with a bolus of contrast media $(40 \mathrm{ml}) 10$ minutes after CTA in our routine BD protocol. CTP scan was performed between the level of internal carotid artery bifurcation and superior edge of lateral ventricles with a section thickness of $10 \mathrm{~mm}$ and a total scan time of 45 seconds. ROIs were placed automatically at the anterior cerebral artery for arterial input and superior sagittal sinus for venous outflow. Cerebral blood flow (CBF), cerebral blood volume (CBV), and mean transit time (MTT) maps were acquired by automatic calculation. All images were assessed independently by two radiologists (H.S. and Y.P.) with five years of experience in the evaluation of CTP for BD confirmation. Final decision was made by a consensus if there was a conflict in the results. Readers were blinded to the clinical examination and previous radiological reports in terms of $\mathrm{BD}$ diagnosis. $\mathrm{CBV}$ and $\mathrm{CBF}$ maps were evaluated qualitatively, and complete absence of perfusion in the cerebral parenchyma was accepted as cerebral circulatory arrest.

\section{Statistical analysis}

Statistical analysis was made using SPSS software version 21.0 (IBM). The Mann-Whitney $U$ test was used to compare GM and WM density and the GM/WM density ratio of $\mathrm{BD}$ patients with the normal control group. Receiver operating characteristic (ROC) curves were analysed to identify the cut-off value of the GM/WM density ratio for the prediction of $\mathrm{BD}$ as well as calculating the sensitivity and specificity. Inter-rater variability was evaluated by using interclass correlation coefficient (ICC). $r$ values including the range from 0 to 1.00 were described as follows: $<0.40$, poor; 0.41-0.60, moderate; 0.61-0.80, good; $\geq 0.81$, excellent agreement. A $p$ value of less than 0.05 was considered statistically significant.

\section{Results}

Seventy-seven patients clinically diagnosed as BD (36 female, 41 male; mean age, $43.84 \pm 23.25$ years; range 0-80 years) and 77 age- and sex-matched control subjects (36 female, 41 male; mean age, $43.92 \pm 23.07$ years; range 
Table I. Comparison of density values and ratio at each level between the patient and control groups

\begin{tabular}{|c|c|c|c|}
\hline Level & Patient group & Control group & $p$-value \\
\hline \multicolumn{4}{|l|}{ Basal ganglia } \\
\hline BG grey matter & $32.08 \pm 4.27$ & $42.05 \pm 5.79$ & $<0.001$ \\
\hline Caudate nucleus & $31.46 \pm 4.59$ & $43.26 \pm 6.90$ & $<0.001$ \\
\hline Putamen & $32.70 \pm 4.60$ & $40.84 \pm 5.18$ & $<0.001$ \\
\hline BG white matter & $27.29 \pm 3.11$ & $35.26 \pm 6.47$ & $<0.001$ \\
\hline Corpus callosum & $27.30 \pm 3.32$ & $36.09 \pm 7.34$ & $<0.001$ \\
\hline Posterior limb of internal capsule & $27.28 \pm 3.71$ & $34.42 \pm 6.21$ & $<0.001$ \\
\hline GM/WM density ratio & $1.17 \pm 0.12$ & $1.20 \pm 0.11$ & 0.069 \\
\hline \multicolumn{4}{|l|}{ Centrum semiovale } \\
\hline Grey matter & $33.26 \pm 4.89$ & $46.82 \pm 9.17$ & $<0.001$ \\
\hline White matter & $29.33 \pm 3.84$ & $35.37 \pm 7.36$ & $<0.001$ \\
\hline GM/WM density ratio & $1.13 \pm 0.14$ & $1.33 \pm 0.14$ & $<0.001$ \\
\hline \multicolumn{4}{|l|}{ High convexity } \\
\hline Grey matter & $35.67 \pm 5.24$ & $47.60 \pm 9.82$ & $<0.001$ \\
\hline White matter & $32.60 \pm 4.63$ & $36.22 \pm 8.21$ & 0.019 \\
\hline GM/WM density ratio & $1.09 \pm 0.11$ & $1.32 \pm 0.12$ & $<0.001$ \\
\hline \multicolumn{4}{|l|}{ Cerebellum } \\
\hline Grey matter & $39.76 \pm 7.57$ & $49.98 \pm 9.15$ & $<0.001$ \\
\hline White matter & $32.09 \pm 5.28$ & $37.01 \pm 7.59$ & $<0.001$ \\
\hline GM/WM density ratio & $1.26 \pm 0.31$ & $1.36 \pm 0.16$ & $<0.001$ \\
\hline \multicolumn{4}{|l|}{ Brainstem } \\
\hline Midbrain & $30.54 \pm 5.37$ & $39.53 \pm 9.14$ & $<0.001$ \\
\hline Pons & $31.22 \pm 6.29$ & $36.80 \pm 5.98$ & $<0.001$ \\
\hline Medulla oblongata & $27.82 \pm 9.58$ & $38.76 \pm 6.70$ & $<0.001$ \\
\hline
\end{tabular}

Data refer to mean value \pm standard deviation. $B G$ - basal ganglia, GM - grey matter, WM - white matter

$0-83$ years) were included in the study. The initial causes of coma were intracranial haemorrhage $(n=36,46.7 \%)$, high-energy trauma $(n=11,14.2 \%)$, cardiac arrest $(n=10$, $12.9 \%)$, postoperative clinical conditions $(n=8,10.3 \%)$, intoxication $(n=6,7.7 \%)$, ischaemic stroke $(n=3,3.8 \%)$, infection $(n=2,2.5 \%)$, and asphyxia $(n=1,1.2 \%)$.

In a comparison between the patient and control groups, WM and GM densities at the level of BG (caudate nucleus, putamen, corpus callosum, and posterior limb of internal capsule), CS, HC, BS (midbrain, pons, and medulla oblongata), and cerebellum were significantly lower in BD patients ( $p=0.019$ for $\mathrm{HC}-\mathrm{WM}, p<0.001$ for other areas). The GM/ WM density ratio of CS, HC, and cerebellum were found to be significantly lower in $\mathrm{BD}$ patients ( $p<0.001$ for all levels), but there was no difference of GM/WM density ratio at the level of BG between the two groups $(p=0.069)$ (Table 1 ).

ROC analysis of the GM/WM density ratio for predicting BD showed that the highest value of area under curve (AUC) was found to be 0.907 at the level of HC $(\mathrm{AUC}=0.907, \mathrm{CI}: 0.858-0.956)$. AUC values of CS, BG, and cerebellum level were identified as $0.837,0.585$, and 0.670 , respectively (Figure 3 ). When a cut-off value of
GM/WM density ratio at HC level was determined as 1.25 , sensitivity and specificity values in predicting $\mathrm{BD}$ were $90 \%$ and $73 \%$, respectively.

When CTP was assessed alone in patients with BD, $72 / 77$ patients were interpreted as BD with sensitivity of 93\%. The addition of CTP to the evaluation of the GM/ WM density ratio of the $\mathrm{HC}$ level increased the sensitivity in predicting BD to $98 \%$ (Figure 4 ).

Inter-rater agreement of the radiologists in evaluating the density measurement of GM and WM from specified regions was found to be excellent at the level of BG for both GM and WM ( $r=0.869$ and 0.817 , respectively); at the level of CS WM $(r=0.894)$ and the level of HC WM $(r=0.830)$. The lowest agreement was detected at the level of cerebellum WM, which was moderate $(r=0.522)$. All values for each level are shown in Table 2.

\section{Discussion}

In our study, the value of density measurement and the GM/WM density ratio in predicting BD were evaluated, and the GM-WM densities of each of 23 areas and the 


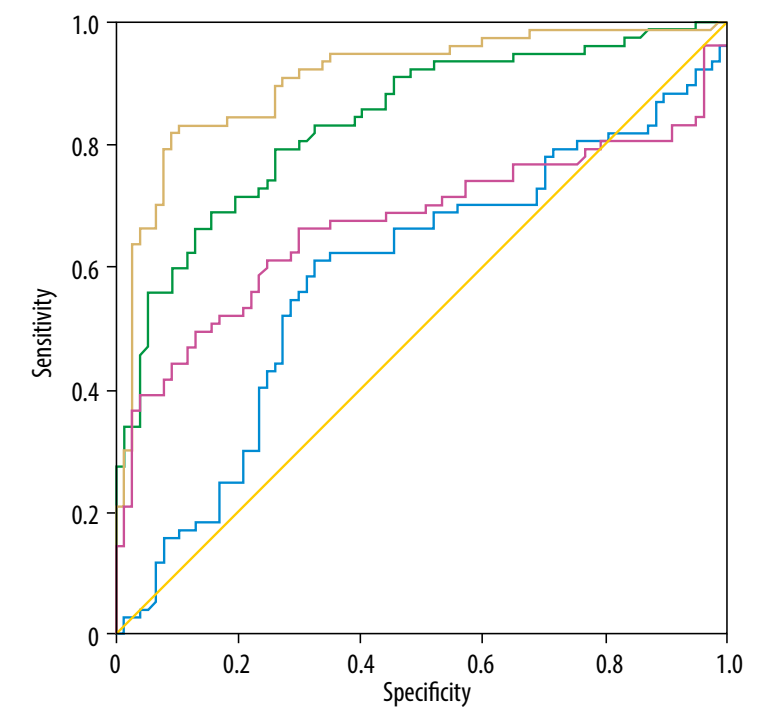

- BG ratio - CS ratio - HCL ratio - Cerebellum ratio - Reference line

BG - basal ganglia, CS - centrum semiovale, HCL - high convexity level

Figure 3. Receiver operating curve analysis of grey matter/white matter density ratio at each level for predicting brain death

GM/WM density ratio of each level except for BG were found to be significantly lower in $\mathrm{BD}$ patients compared to the control group ( $p=0.019$ for HC-WM, $p<0.001$ for other areas). A cut-off value of GM/WM density ratio at the $\mathrm{HC}$ level was determined as 1.25 with a sensitivity of $90 \%$ and specificity of $73 \%$ for the prediction of BD. The sensitivity increased to $98 \%$ when CTP was used as an adjunct to density measurement.

Diffuse decrease in the cortical GM density has been defined as one of the CT findings of cerebral hypoperfusion [21]. This appearance could be explained by cytotoxic oedema, which occurs as a result of the disruption of the sodium-potassium pump in anoxic cells and is known to be more prominent in GM due to the high metabolic demand [21]. In our study, this finding was assessed by perform- ing various HU measurements from brain parenchyma in brain-dead patients, and the GM density was found to be significantly low when compared to the control group at each level. Low density values in GM have also been shown in previous studies consisting of comatose or brain-dead patient groups $[8,11,17,19]$. However, there is a discrepancy between studies regarding the results of WM density. In many studies performed in comatose patients after cardiac arrest, WM values were not found to be statistically significant [14-17,22,23], whereas an increase in WM density compared to the control group was recorded in one study [11]. In our study, WM density was found to be significantly lower in patients with $\mathrm{BD}$, which was also shown by Vigneron et al. [19]. This situation could be explained by the difference in characteristics of patient populations and possibly the timing of the CT scan.

Quantitative measurements on CT imaging have been commonly studied to evaluate the distinction of vegetative state-death or good-poor neurologic outcome after cardiac arrest $[8,11,12,17,23,24]$. The first study of the use of GM/WM density ratio to assess the prognosis of comatose patients after cardiac arrest was conducted by Torbey et al., in which the cut-off ratio was found to be 1.18 [11]. They indicated that values below this rate at the BG level were $100 \%$ predictive for death. In a meta-analysis involving 1150 patients from 10 studies, it was concluded that the GM/WM ratio had a high prognostic value in predicting a poor neurologic outcome in patients after cardiac arrest in the early period [25]. They reported that the cut-off values of seven studies at BG level were between 1.10 and 1.24 [25]. In a study consisting of 15 brain-dead patients conducted by Vigneron et al., the cut-off value was determined as 1.21 at basal ganglion level, but in their study the sensitivity was not stated [19]. In our study, with a greater patient population, it was found that values below 1.25 in the GM/WM ratio at the $\mathrm{HC}$ level could be useful in predicting $\mathrm{BD}$ with high sensitivity. Also, unlike in the above
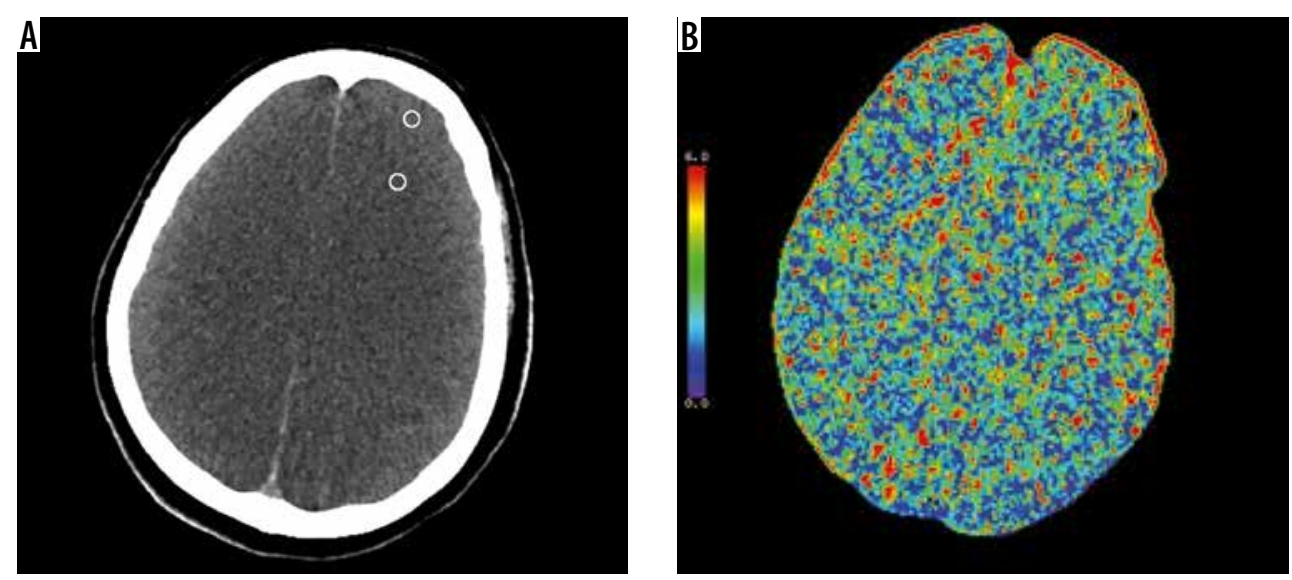

Figure 4. A 54-year-old female patient clinically diagnosed as brain death. A) Unenhanced axial computed tomography image at high convexity level shows diffuse cerebral edema and loss of differentiation between gray and white matter. The attenuation values of grey matter (GM) and white matter (WM) were 37 and $34 \mathrm{HU}$, respectively. GM/WM ratio was found as 1.08 which was below 1.25. (The regions where regions of interests are placed in gray and white matter were shown schematically with a white circle.) B) Cerebral blood volume map demonstrates the absence of cerebral perfusion which was compatible with brain death. (Cerebral blood flow and mean transit time maps not shown) 
Table 2. Inter-rater agreement results in evaluating the parenchymal density measurement

\begin{tabular}{|l|c|c|c|}
\multirow{2}{*}{ Level } & \multicolumn{3}{|c|}{ Parenchymal density measurement } \\
\cline { 2 - 4 } & Reader 1 & Reader 2 & ICC (CI) \\
\hline BG-GM & $32.13 \pm 4.27$ & $30.69 \pm 3.67$ & $0.869(0.692-0.933)$ \\
\hline BG-WM & $27.29 \pm 3.13$ & $28.30 \pm 3.17$ & $0.817(0.674-0.892)$ \\
\hline CS-WM & $33.26 \pm 4.89$ & $34.06 \pm 4.70$ & $0.737(0.587-0.832)$ \\
\hline HC-GM & $29.33 \pm 3.84$ & $30.07 \pm 3.57$ & $0.894(0.824-0.935)$ \\
\hline HC-WM & $35.67 \pm 5.24$ & $34.37 \pm 4.58$ & $0.808(0.685-0.881)$ \\
\hline Cerebellum-GM & $32.60 \pm 4.63$ & $30.77 \pm 4.02$ & $0.830(0.600-0.914)$ \\
\hline Cerebellum-WM & $39.76 \pm 7.57$ & $36.26 \pm 4.50$ & $0.522(0.211-0.705)$ \\
\hline Midbrain & $32.09 \pm 5.28$ & $30.96 \pm 3.56$ & $0.669(0.481-0.789)$ \\
\hline Pons & $30.55 \pm 5.37$ & $30.19 \pm 4.58$ & $0.738(0.588-0.834)$ \\
\hline Medulla oblongata & $31.22 \pm 6.29$ & $30.75 \pm 5.37$ & $0.573(0.325-0.729)$ \\
\hline
\end{tabular}

Data of reader 1 and reader 2 refer to mean value \pm standard deviation. CI - 95\% confidence interval, ICC - interclass correlation coefficient, BG - basal ganglia, CS - centrum semiovale, HC - high convexity, GM - grey matter, WM - white matter

study, the density measurement at this level was found to have very close sensitivity values (90\%) compared to CTP (93\%) obtained in the same session. We suggest that measurements from this localisation could provide more reliable results in such a heterogeneous patient population with comorbid diseases and/or intracranial haemorrhage.

There are a number of studies that have investigated the contribution of CTP to CTA in confirmation of BD $[2-4,26]$. To our knowledge, this is the first study on evaluation of density measurements on unenhanced CT with the added value of CTP in predicting BD. According to the pattern of BD proposed by Palmer and Bader, a lack of cerebral blood flow is seen when intracranial pressure exceeds mean arterial pressure [1]. As a result, cerebral circulatory arrest leads to cytotoxic injury and subsequent irreversible neuronal damage. For determination of the absence of cerebral perfusion, CTP could be a suitable method in patients clinically diagnosed as BD. While the sensitivity of CTP was found to be $93 \%$ in our study, this value reached $98 \%$ with the addition of the GM/WM density ratio from the $\mathrm{HC}$ level. According to this result, we think that BD could be predicted with $98 \%$ sensitivity by performing a combined protocol of unenhanced CT scan and CTP with a $40 \mathrm{ml}$ contrast media without CTA. However, this result needs to be supported by further studies involving more $\mathrm{BD}$ patients and a separate group of coma and intensive care patients as the control group.

Our study has several limitations. First, the timing of CT scan was not the same for all brain-dead patients. This condition might affect the attenuation of brain parenchyma among patients. However, in a study conducted by Metter et al., no significant relationship was found between the time from arrest to CT and the attenuation values in patients with cardiac arrest [8]. In another study, Scheel et al. reported that there was a gradual decrease in the median GM/WM density ratio value in CTs taken at six hours, 6-24 hours, and 24 hours, but there was no statistically significant difference between the three groups [23]. Contrarily, Wu et al. found a significant decrease in putamen density on CT taken at 24-72 hours compared to the first 24 hours [24]. However, as far as we know, there is no study on the alteration of attenuation over time after the diagnosis of BD. Second, in some patients, haematoma, herniation, shift, and artifacts passing through brainstem level caused difficulty in measuring the density. In order to determine the effect of this limitation on the results, interobserver reliability was evaluated and found to be in good or excellent agreement, except for cerebellum GM, midbrain, and pons levels, which is possibly due to the beam hardening artifacts passing through the posterior fossa. Third, CTP was performed after CTA because CTP has not yet been proven as a valid method in BD diagnosis. Contrast stasis in vessels may potentially create a limitation for the CTP. We attempted to minimise this limitation by allowing a waiting period of 10-15 minutes after CTA; a longer waiting period could not be performed because this patient group had to be followed under intensive care conditions.

\section{Conclusions}

The GM/WM density ratio at the HC level on unenhanced CT scan may be a useful finding to predict BD. The addition of CTP promotes this prediction by increasing the sensitivity. The combination of unenhanced CT and CTP might be reasonably acceptable by using lower amount of intravenous contrast media for BD patients who are potential candidates as donors for organ transplantation.

\section{Conflict of interest}

The authors report no conflict of interest. 


\section{References}

1. Heran MK, Heran NS, Shemie SD. A review of ancillary tests in evaluating brain death. Can J Neurol Sci 2008; 35: 409-419.

2. Sawicki M, Solek-Pastuszka J, Chamier-Cieminska K, et al. Computed tomography perfusion is a useful adjunct to computed tomography angiography in the diagnosis of brain death. Clin Neuroradiol 2019; 29: 101-108.

3. Shankar JJ, Vandorpe R. CT perfusion for confirmation of brain death. AJNR Am J Neuroradiol 2013; 34: 1175-1179.

4. Escudero D, Otero J, Marqués L, et al. Diagnosing brain death by CT perfusion and multislice CT angiography. Neurocrit Care 2009; 11: 261-271.

5. Yoshikai T, Tahara T, Kuroiwa T, et al. Plain CT findings of brain death confirmed by hollow skull sign in brain perfusion SPECT. Radiat Med 1997; 15: 419-424.

6. Kandiah P, Ortega S, Torbey MT. Biomarkers and neuroimaging of brain injury after cardiac arrest. Semin Neurol 2006; 26: 413-421.

7. Morimoto Y, Kemmotsu O, Kitami K, et al. Acute brain swelling after out-of-hospital cardiac arrest: pathogenesis and outcome. Crit Care Med 1993; 21: 104-110.

8. Metter RB, Rittenberger JC, Guyette FX, et al. Association between a quantitative CT scan measure of brain edema and outcome after cardiac arrest. Resuscitation 2011; 82: 1180-1185.

9. Inamasu J, Miyatake S, Suzuki M, et al. Early CT signs in out-of-hospital cardiac arrest survivors: temporal profile and prognostic significance. Resuscitation 2010; 81: 534-538.

10. Inamasu J, Miyateke S, Nakatsukasa M, et al. Loss of gray-white matter discrimination as an early CT sign of brain ischemia/hypoxia in victims of asphyxial cardiac arrest. Emerg Radiol 2011; 18: 295-298.

11. Torbey MT, Selim M, Knorr J, et al. Quantitative analysis of the loss of distinction between gray and white matter in comatose patients after cardiac arrest. Stroke 2000; 31: 2163-2167.

12. Lee BK, Jeung KW, Song KH, et al. Prognostic values of gray matter to white matter ratios on early brain computed tomography in adult comatose patients after out-of-hospital cardiac arrest of cardiac etiology. Resuscitation 2015; 96: 46-52.

13. Hong JY, Lee DH, Oh JH, et al. Grey-white matter ratio measured using early unenhanced brain computed tomography shows no correlation with neurological outcomes in patients undergoing targeted temperature management after cardiac arrest. Resuscitation 2019; 140: 161-169.

14. Kim SH, Choi SP, Park KN, et al. Early brain computed tomography findings are associated with outcome in patients treated with therapeutic hypothermia after out-of-hospital cardiac arrest. Scand J Trauma Resusc Emerg Med 2013; 19: 21-57.
15. Gentsch A, Storm C, Leithner C, et al. Outcome prediction in patients after cardiac arrest: a simplified method for determination of gray-white matter ratio in cranial computed tomography. Clin Neuroradiol 2015; 25: 49-54.

16. Wang GN, Chen XF, Lv JR, et al. The prognostic value of gray-white matter ratio on brain computed tomography in adult comatose cardiac arrest survivors. J Chin Med Assoc 2018; 81: 599-604.

17. Choi SP, Park HK, Park KN, et al. The density ratio of grey to white matter on computed tomography as an early predictor of vegetative state or death after cardiac arrest. Emerg Med J 2008; 25: 666-669.

18. Callaway CW, Donnino MW, Fink EL, et al. Part 8: post-cardiac arrest care: 2015 American Heart Association guidelines update for cardiopulmonary resuscitation and emergency cardiovascular care. Circulation 2015; 132: S465-482.

19. Vigneron C, Labeye V, Cour M, et al. Gray matter-white matter de-differentiation on brain computed tomography predicts brain death occurrence. Transplant Proc 2016; 48: 1893-1897.

20. Archil-Rincon LM, Del Carmen Garcia-Blanco M, Roldan-Valadez E. Diagnostic performance of CT densities in selected gray-and white-matter regions for the clinical diagnosis of brain death: a retrospective study in a tertiary-level general hospital. Eur J Radiol 2018; 108: 66-77.

21. Kjos BO, Brant-Zawadzki M, Young RG. Early CT findings of global central nervous system hypoperfusion. AJR Am J Roentgenol 1983; 141: 1227-1232.

22. Yamamura H, Kaga S, Kaneda K, et al. Head computed tomographic measurement as an early predictor of outcome in hypoxic-ischemic brain damage patients treated with hypothermia therapy. Scand J Trauma Resusc Emerg Med 2013; 21: 37.

23. Scheel M, Storm C, Gentsch A, et al. The prognostic value of graywhite-matter ratio in cardiac arrest patients treated with hypothermia. Scand J Trauma Resusc Emerg Med 2013; 21: 23.

24. Wu O, Batista LM, Lima FO, et al. Predicting clinical outcome in comatose cardiac arrest patients using early noncontrast computed tomography. Stroke 2011; 42: 985-992.

25. Na MK, Kim W, Lim TH, et al. Gray matter to white matter ratio for predicting neurological outcomes in patients treated with target temperature management after cardiac arrest: a systematic review and meta-analysis. Resuscitation 2018; 132: 21-28.

26. Bohatyrewicz R, Sawicki M, Walecka A, et al. Computed tomographic angiography and perfusion in the diagnosis of brain death. Transplant Proc 2010; 42: 3941-3946. 